\title{
Linx
}

Revue des linguistes de l'université Paris X Nanterre

$51 \mid 2004$

Théories de l'écriture et pratiques scolaires

\section{Quelques spécificités des approches didactiques de l'écriture}

Yves Reuter

\section{(2) OpenEdition}

Journals

Édition électronique

URL : http://journals.openedition.org/linx/165

DOI : $10.4000 / \operatorname{linx} .165$

ISSN : 2118-9692

Éditeur

Presses universitaires de Paris Nanterre

Édition imprimée

Date de publication : 1 décembre 2004

Pagination : 41-54

ISSN : 0246-8743

\section{Référence électronique}

Yves Reuter, "Quelques spécificités des approches didactiques de l'écriture », Linx [En ligne],

51 | 2004, mis en ligne le 27 janvier 2011, consulté le 19 avril 2019. URL : http://

journals.openedition.org/linx/165 ; DOI : 10.4000/linx.165

Département de Sciences du langage, Université Paris Ouest 


\title{
Quelques spécificités des approches didactiques de l'écriture
}

\author{
Yves Reuter \\ Université Charles-de-Gaulle - LILLE III \\ Équipe de recherche THÉODILE (E.A. 1764)
}

Je souhaite dans cet article tenter de répondre aux interpellations de la journée d'étude "Quand les pratiques scolaires interrogent les théories et modèles de l'écriture » quant au statut de la didactique du français, à ses modes de construction des formalisations de l'écriture et à ses relations avec les autres disciplines qui s'en préoccupent. A cette fin, je développerai essentiellement les quatre points suivants : la définition de la didactique du français, les causes possibles de certains malentendus dans les rapports avec les autres disciplines, les modalités des collaborations possibles et enfin, en relation avec les deux questions précédentes, quelques spécificités des démarches méthodologiques en didactique ${ }^{1}$.

\section{Définir la didactique du français ${ }^{2}$}

La didactique du français peut se définir, au moins en première approche, comme la discipline de recherche qui a pour objet l'analyse des savoirs, savoir-faire et des pratiques d'enseignement-apprentissage de la discipline «français», essentiellement au sein de l'appareil scolaire.

Cette définition, relativement consensuelle ${ }^{3}$, implique une double différence dont il importe de prendre la mesure :

- différence avec les pratiques d'enseignement-apprentissage et toute forme de contrôle, de prescription ou d'ingénierie ;

\footnotetext{
${ }^{1}$ Par souci d'économie, je remplacerai souvent l'expression « didactique du français » par le terme de didactique (au singulier) ; didactiques renvoyant aux différentes didactiques (des mathématiques, de l'histoire...).

${ }^{2}$ Je reprends très brièvement ici des propositions déjà formulées dans Brassart et Reuter 1992 ou Reuter 1994 a.

${ }^{3}$ Voir, par exemple, Halté 1992.
} 
- différence avec deux ensembles théoriques, intéressés indirectement par l'objet construit par la didactique : celui des théories de référence possibles des savoirs et savoir-faire (langue, texte, discours, littérature, lecture, écriture...) en tant qu'elles ne se préoccupent pas centralement de l'enseignement-apprentissage ; celui des théories de référence possibles des modes d'enseignement et d'apprentissage, en tant qu'elles ne se préoccupent pas centralement des contenus.

Pour le dire nettement, c'est de recherches dont il s'agit et ces recherches articulent nécessairement contenus spécifiques - qui définissent les différentes didactiques - et enseignement-apprentissages.

Cependant, ces ruptures ne sont pas absolues, excluant toute possibilité de dialectique entre les pôles distingués. Ainsi, le clivage instauré entre recherches et pratiques, s'il est fondateur et nécessaire pour garantir le statut «scientifique » des didactiques, n'exclut pas la prise en compte de ce que j’ai appelé un borizon praxéologique. Il me semble en effet qu'une partie de la spécificité des didactiques réside dans leur pertinence sociale. Celle-ci tient tout autant à leur prise en compte des fonctionnements et des dysfonctionnements disciplinaires enregistrés sur le «terrain » qu'à leur contribution possible à leur analyse et à la construction de pistes d'amélioration potentielles.

Cela à trois conditions cependant : le caractère théoriquement et empiriquement fondé des propositions effectuées, leur évaluation afin d'en appréhender les intérêts et les limites, leur inscription à titre d'apports au sein d'une palette de possibles dont il appartient aux prescripteurs, aux formateurs et aux praticiens de s'emparer ou non. Ces trois conditions tracent à mon sens nettement la frontière entre recherches et interventions. Elles ouvrent un espace possible pour la gestion de la tension entre le pôle de l'abstention, difficilement tenable de manière absolue au vu des objets étudiés ${ }^{4}$, et celui de l'intervention qui comporte inéluctablement les dérives connues et éprouvées dans le passé du scientisme, de l'applicationnisme ou du militantisme (voir infra 2.2).

Complémentairement, le clivage instauré avec les théories des objets disciplinaires et celles de l'enseignement et / ou de l'apprentissage, s'il est fondateur et nécessaire pour distinguer la didactique de disciplines proches, n'implique pas leur expulsion, puisque les objets de la didactique n'existent qu'en tant qu'ils articulent ces deux dimensions. La question - et les dissensus qu'elle suscite chez les didacticiens réside plutôt dans les dominantes retenues quant aux modes d'articulation: accent plutôt porté sur l'un ou l'autre pôle, et à l'intérieur de chacun de ces pôles, place accordée à telle ou telle discipline de recherche (voire à tel ou tel courant) ou part conférée à la multiréférentialité...

Ces éléments, trop succincts, de définition entraînent au moins deux conséquences sur le terrain de l'écriture :

- à l'instar de toute autre discipline de recherche, la didactique du français ne s'intéresse pas à l'écriture «en général » mais à l'écriture en tant qu'elle s'enseigne et s'apprend en accordant une attention particulière à ce qui active ou entrave son développement ;

\footnotetext{
4 et au fait que les didactiques seraient autrement absolument tributaires des décisions institutionnelles quant au choix des contenus sur lesquels réfléchir.
} 
- elle effectue cela, en inscrivant en son sein, une interdisciplinarité structurelle, dans la mesure où elle ne peut a priori se priver d'aucun référent théorique susceptible de l'éclairer quant aux objectifs et aux pratiques retenues par l'école pour enseigner l'écriture, quant aux choix et aux mécanismes de transposition didactique mis en place (Chevallard 1985), quant aux relations entre pratiques scolaires et extrascolaires, quant aux fonctionnements et aux dysfonctionnements, à leur analyse, à leurs sources possibles et aux remédiations envisageables.

Reste encore, et ce n'est pas là sa moindre tâche, à reconstruire ces référents en une configuration théoriquement plausible et pertinente...

\section{Des relations difficiles avec les autres disciplines}

Le statut et les caractéristiques de la didactique du français tels que je viens de les énoncer a pu entrainer, parfois, des rapports difficiles avec les autres disciplines ou, du moins avec certains de leurs représentants, souvent d'ailleurs ceux qui, par méconnaissance ${ }^{5}$, la confondaient avec l'ingénierie. Ces problèmes peuvent peut-être être catégorisés à partir de trois questions ${ }^{6}:$ celle de la défense du territoire, celle de la critique de l'applicationnisme, et celle de la confrontation interdisciplinaire.

\subsection{La défense du territoire}

Toute discipline veille jalousement, institutionnellement et épistémologiquement, sur le territoire qu'elle a souvent conquis de haute lutte et qui lui confère en retour son autorité. Or, de ce point de vue, l'émergence récente de la didactique n'a pu que provoquer des remous puisqu'elle prétend, d'un côté, avoir des éléments à apporter sur les savoirs (langue, textes, discours, littérature...) et les savoir-faire (lecture, écriture, parole, écoute, compréhension, interprétation...) impliqués par le français ${ }^{7}$ et, de l'autre, avoir des choses à dire sur l'enseignement et les apprentissages en tant qu'ils sont référés à des contenus disciplinaires qui les spécifient ${ }^{8}$.

Conséquemment, la didactique opère un coup de force face à certaines conceptions. De discipline ancillaire, tenue de s'appuyer sur les disciplines dites de référence, elle transforme celles-ci en disciplines contributoires dans le cadre de son projet de connaissance.

\footnotetext{
${ }^{5}$ Cette méconnaissance se lit au travers de la conjonction entre des définitions hasardeuses (confondant souvent didactique et pratiques d'enseignement) et une absence de toute référence bibliographique propre à la didactique.

${ }^{6}$ Qui sont, de fait, comme j'espère pouvoir le montrer, trois aspects d'une même position.

${ }^{7}$ Dans la mesure par exemple où, d'un côté, il lui est nécessaire de confronter des approches issues de diverses origines théoriques et, où d'un autre côté, les difficultés d'enseignement-apprentissage sont susceptibles d'éclairer des aspects problématiques ou des fonctionnements moins connus des pratiques et des objets langagiers (de manière proche de ce que peuvent éclairer des approches dans le domaine du développement ou de la pathologie du langage...).

8 À la différence de conceptions et de recherches qui ne les envisagent que de manière très générale.
} 
C'est ainsi le cas lorsqu'elle construit des modèles didactiques', de l'écriture, étayés notamment sur les théories qui éclairent ses fonctionnements et ses dysfonctionnements, sur l'histoire de son enseignement et de ses effets, sur l'analyse des difficultés des élèves, sur les théories des objets langagiers, cela afin de spécifier les principales dimensions de l'écriture en tant qu'elle s'enseigne et s'apprend, de préciser ses lieux problématiques, de cerner les choix scolaires (en termes d'objectifs, de stratégies, de pratiques...), d'appréhender les causes possibles des difficultés des apprenants.

La didactique est ainsi amenée, en tant que discipline d'interactions, à déconstruire les constructions des autres disciplines, à sélectionner certaines de leur composantes et à les recomposer avec d'autres, en fonction de ses finalités. C'est notamment le cas, lorsqu'un modèle de l'écriture articule des opérations cognitives (quelque peu remodelées) avec la notion de pratiques ${ }^{10}$ et des formalisations d'objets langagiers ${ }^{11}$.

Je remarquerai cependant que, ce qui peut apparaitre aux yeux de certains comme un coup de force, ne l'est qu'à leur supposer un monopole de discours sur les terrains explorés par la didactique. Si tel n'est pas le cas, on se retrouve alors dans un cas de figure classique dans les sciences humaines: sur des territoires voisins, chaque discipline use des autres en fonction de son projet de connaissance.

Cela n'exclut nullement, mais fonde, les conditions de possibilité d'une vigilance épistémologique :

- quant à la spécificité de ce projet de connaissance qui se définit, au moins en grande partie, contrastivement;

- quant à la validité des constructions effectuées, notamment en ce qui concerne l'intégration des «emprunts» qui ne sauraient se réduire à une simple juxtaposition (ou à une caution partielle).

\subsection{La critique de l'applicationnisme}

À cette première dimension susceptible d'expliquer certaines difficultés, s'ajoute une seconde source de tensions engendrée par l'analyse critique effectuée par les didacticiens de positions d'autres chercheurs face au terrain scolaire. Ces positions peuvent se résumer en la croyance que telle science pourrait (ou devrait) avoir un rôle de pilote pour guider les pratiques d'enseignement dans la mesure où il suffirait d'appliquer ses savoirs pour engendrer des effets positifs sur le terrain scolaire.

\footnotetext{
${ }^{9}$ Sur la notion de modèle didactique, en débat dans le champ didactique, voir d'un côté De Pietro et Schneuwly (2003), Dolz et Schneuwly (1998) ou Enjeux (1996 / 1997) et, de l'autre Reuter 1996, 2000 ou 2001

${ }^{10}$ Qui réinscrit les dimensions psychoaffectives et socioculturelles des sujets et des activités, la diversité des situations, les variations de normes, ainsi que la matérialité des outils et des supports.

${ }^{11}$ Sur les problèmes théoriques que de telles articulations soulèvent, voir mes remarques dans Reuter 1996 et 2002.
} 
Cette position, quelque peu caricaturée $i^{1} i^{12}$, n'a cessé de générer des effets connus sous le nom d'applicationnisme et qui ont concerné, par exemple, la grammaire structurale ou la narratologie. Pour n'en prendre que trois exemples plus récents, j'évoquerai ainsi la vogue du modèle «Hayes et Flower» de l'écriture, celle des typologies de textes passées du statut d'outil heuristique à celui de prescription dans les pratiques scolaires ${ }^{13}$, ou celle de la notion de révision qui, dérivée des travaux de psychologie cognitive et de la génétique textuelle, a engendré des exercices formels ${ }^{14}$ réduisant conséquemment ses intérêts potentiels (ouvrir à une conception plus dynamique de l'écriture, analyser les multiples modalités de ses opérations constitutives et les formes possibles des procédures qui l'actualisent...).

Cette conception s'appuie ainsi sur des images discutables de la science (détentrice de vérités ${ }^{15}$ ) et des relations entre recherches et pratiques scolaires (les unes devant servir de guides aux autres ${ }^{16}$ ). Elle méconnait de surcroît l'intérêt d'un espace théorique spécifique pensant et reconfigurant les apports possibles des disciplines en relation avec l'enseignement-apprentissage. Elle ne peut dès lors que se sentir menacée par la mise en cause de son statut de référent, ainsi que par l'analyse des pratiques qui s'emparent de ses propositions et des effets qu'elles suscitent ${ }^{17}$.

\subsection{La confrontation interdisciplinaire}

Une troisième dimension participe encore des malentendus évoqués. Il s'agit de la confrontation mise en œuvre par la didactique et tant que discipline d'interactions. Dans certains cas en effet, par les confrontations qu'elle implique, elle est amenée à mettre au jour des discordances théoriques entre disciplines différentes.

J'en prendrai ici trois exemples afin de préciser mon propos. Pour certaines disciplines, suivant en cela la tradition socio-scolaire, l'écriture est une activité individuelle (voire solitaire). Pour d'autres, en revanche, notamment pour celles qui s'intéressent aux pratiques professionnelles ${ }^{18}$, elle n'est jamais réellement individuelle (par ses conditions d'exercice, par le dialogue qu'elle instaure avec les autres, par l'intertextualité qu'elle met en œuvre...). D'autre part, certaines disciplines formalisent l'écriture, sur un mode abstrait (que j'ai appelé par ailleurs celui de «l'activité ») qui unifie l'écriture, via un noyau d'invariants et des opérations de décontextualisation. À

\footnotetext{
12 Caricaturée dans la mesure où elle n'est jamais exposée aussi explicitement. En revanche, la poursuite effrénée de certaines positions institutionnelles et les discours prescriptifs qui s'ensuivent, témoignent de l'existence de cette position...

13 Il s'agit alors, sur le modèle de la grammaire «traditionnelle », d'enseigner aux élèves à reconnaittre des types « purs ». Pour une critique de cette position dans le cas de la description, voir Reuter 2000.

14 Exercices en deux temps («Ecrivez un texte »; « Réécrivez le ») sans articulation avec la situation de communication, le projet de l'élève, des analyses précises des fonctionnements et dysfonctionnements...

${ }^{15}$ Ignorant par là même les cadavres théoriques qui jonchent l'histoire des sciences.

16 Niant par là même l'espace de responsabilité des maîtres.

${ }^{17}$ Parfois à leur corps défendant d'ailleurs.

18 Voir, par exemple, Borzeix et Fraenckel, eds, 2001 ou Delcambre 1997.
} 
l'inverse, d'autres disciplines insistent, via la notion de pratiques $^{19}$ sur la diversité d'écritures toujours contextualisées.

Enfin, je prendrai comme troisième exemple, sensiblement différent, celui de l'activité métacognitive, considérée par certains comme une compétence technique et par d'autres comme une disposition socialement construite et inégalement partagée, susceptible d'expliquer, au moins en partie, l'échec scolaire lors de l'entrée dans l'écrit. (Lahire 1993).

On voit bien que cette mise en confrontation de continents théoriques qui, autrement, vivraient sereinement dans la relative ignorance de leurs divergences attribuables, légitimement, à des perspectives et à des objets différents, peut susciter un sentiment d'irritation dans la mesure où elle interroge des postulats méthodologiques ou des thèses « fortes », les réduisant à des hypothèses ou des points de vue, et où elle relativise, par voie de conséquence, toute possibilité «mécanique » de transfert sur le terrain scolaire.

\section{Quelques fondements des collaborations possibles}

Les difficultés évoquées n'empêchent nullement des collaborations, effectives depuis de nombreuses années déjà, notamment sur le terrain de l'écriture ${ }^{20}$. Ces collaborations peuvent prendre des formes multiples. J'en privilégierai ici trois parmi celles qui me semblent les plus fructueuses, construites à partir de la confrontation entre les modes de construction du social, du repérage de «zones floues», et de la réflexion sur les discordances théoriques.

\subsection{Confronter les modes de construction $d u$ social}

La première piste de travail que je mentionnerai concerne le découpage et l'articulation des espaces sociaux. En effet, une caractéristique importante de la didactique est de mener ses recherches sur l'espace scolaire, ou à partir de l'espace scolaire, distinguant donc celui-ci des espaces extrascolaires. Mais il lui est cependant indispensable de connaitre ceux-ci pour traiter certaines questions fondamentales, parmi lesquelles les spécificités des sujets et des pratiques scolaires, la connaissance des élèves (de leurs pratiques, de leurs représentations, de leurs compétences...), la congruence ou la discordance entre pratiques scolaires et pratiques extrascolaires (ainsi que leurs effets possibles), les pratiques extrascolaires (ici d'écriture) prises comme références ou comme cibles par les pratiques scolaires ${ }^{21} \ldots$

Il lui est donc nécessaire soit de recourir aux études des autres disciplines sur le domaine extrascolaire, soit d'en mener lorsque ces recherches sont inexistantes ou trop réduites. Cela a été le cas, par exemple, sur les écrits et les pratiques d'écriture

\footnotetext{
${ }^{19}$ Je développe l'opposition entre ces deux modes de formalisation, à propos de la lecture, dans Reuter, 2003.

${ }^{20}$ Voir, par exemple, sur la copie Barré de Miniac, éd, 2000, sur les interactions lecture-écriture, Reuter, ed, 1994, sur la description, Reuter, éd, 1998...

${ }^{21}$ Sur toutes ces questions, voir Repères 2001.
} 
(extra)ordinaires (Dabène 1987, Lidil 1990...) et, plus récemment, sur l'écriture extrascolaire des collégiens (Penloup 1999), permettant ainsi de battre en brèche certaines idées reçues, notamment le faible investissement scriptural des collégiens, et, plus particulièrement, des garçons ou de ceux appartenant aux milieux les moins favorisés.

Mais, en tout état de cause, la confrontation est indispensable pour des raisons qui tiennent :

- aux méthodologies à mettre en œuvre afin de pouvoir croiser déclarations et observations des pratiques, ces dernières posant des problèmes particuliers dans les espaces privés ou professionnels ;

- à la détermination des sens possibles de ce que le chercheur (re) construit : ainsi, les pratiques extrascolaires d'écriture des élèves prennent sens au regard de leur place et de leur statut au sein de configurations de pratiques, excédant l'écriture, structurées et investies différemment selon les catégories de sujets ;

- à la spécification des questions, par exemple, sur les modes d'échange ou de transfert entre pratiques scolaires et extrascolaires, sur leurs points communs et / ou leur différences.

En d'autres termes, la collaboration a des chances d'être fructueuse lorsque les disciplines confrontent leurs questions, leurs découpages et leurs modes d'approche. On voit ainsi que la didactique, centrée sur l'école et procédant à partir d'un découpage de l'espace social en espaces scolaire et extra-scolaire - qui lui est particulier - a besoin de connaissances sur les espaces extrascolaires, élaborés en tenant compte d'autres types de découpages, afin de situer les pratiques, leurs objectifs, leurs relations à ce qui leur est extérieur... Mais, on voit aussi que les autres disciplines ont besoin de la didactique pour mieux percevoir la part du scolaire dans ce qu'elles analysent, mieux comprendre les relations entre espaces sociaux, mieux appréhender les effets ultérieurs des enseignements...

\subsection{L'éclairage de "zones flones"}

La seconde direction de travail concerne des questions, au moins partiellement communes. Tel me semble être le cas des zones langagières « floues $»^{22}$ qui recouvrent des phénomènes difficiles à délimiter strictement, compliqués à analyser et suscitant des jugements flottants (variables) quant à leur acceptabilité.

Il s'agit là d'un phénomène assez fréquent dans les pratiques langagières, écrites ou non, et les évaluations, explicites ou implicites, qui les accompagnent. Sur le terrain des écrits scolaires, je pense par exemple à ces passages de copies que les enseignants délimitent de manière imprécise à l'aide de traits ondulés en marge, en ajoutant ou non une annotation du type «lourd », « mal dit», « maladroit »... Cela peut entraîner des notes basses et des échecs sans que les élèves soient en mesure de comprendre leur problème et d'y remédier dans la mesure où l'enseignant lui-même est relativement démuni, en l'absence de précisions issus des cadres théoriques disponibles. La question est alors de rendre compte de ces jugements en faisant l'hypothèse que, même s'ils sont discutables, ils ne sont pas infondés. On voit bien,

\footnotetext{
${ }^{22}$ Voir, dans ce numéro, la contribution de C. Boré.
} 
dans cette perspective, l'intérêt de recherches communes ou de confrontations théoriques, permettant d'articuler traits linguistiques, caractéristiques des situations, et déclarations des acteurs. C'est, par exemple, ce qu'a réalisé Bernard Daunay, en s'appuyant entre autres sur les travaux de Catherine Fuchs, dans sa thèse sur la paraphrase / le jugement de paraphrase en situation scolaire (Daunay 2002).

Dans ce cadre, de nombreuses recherches seraient d'ailleurs nécessaires : sur la cohérence textuelle et / ou énonciative des écrits d'élèves, sur la répétition (qui, outre des problèmes de seuil, soulève la question de sa stigmatisation ou de sa nécessité selon les situations ou les genres d'écrits ${ }^{23}$ ) et, plus fondamentalement encore sur ce qui est désigné comme dysfonctionnements et sur leurs causes possibles.

Sur ce dernier point, crucial en didactique (Reuter 1994 b, 1998), il me parait d'ailleurs que poser les dysfonctionnements comme traces ${ }^{24}$ permet de confronter de manière heuristique les phénomènes dont elles témoigneraient: la complexité des objets langagiers, les opérations, les procédures, les représentations, la situation, le comportement adopté (prise de risque importante ou non), d'autres pratiques valables dans d'autres champs... Avec l'idée que de cette confrontation peuvent mieux émerger des catégories de causes possibles des dysfonctionnements, non réductibles à de simples manques chez les élèves, ainsi qu'une ouverture plus large de pistes d'enseignement et de remédiation possibles.

Sur ce dernier point, j’ajouterai encore que les modes de catégorisation des dysfonctionnements que permet un modèle didactique de l'écriture, constituent sans doute un critère important d'évaluation de sa pertinence.

\subsection{Penser les discordances théoriques}

La troisième direction de travail que je retiendrai ici, et non la moindre à mes yeux, consiste à reconstruire les discordances inter-théoriques en problèmes à gérer par les théories de la didactique. Je vais tenter de m'en expliquer en reprenant brièvement les exemples que j’avais mentionnés précédemment (cf. 2.3).

Ainsi, la caractérisation de l'écriture en termes d'activité (l'écriture) ou de pratiques (les pratiques d'écriture) renvoie à mon sens à une tension structurante de la didactique. D'un côté, il lui faut une macro-catégorie susceptible de regrouper et d'indexer des pratiques hétérogènes, susceptible aussi de tenir compte de principes constitutifs de l'enseignement scolaire: l'économie des enseignables (afin d'éviter le morcellement, de tenter de penser la transférabilité / traductibilité la plus large de ce qui est enseigné, de permettre la progressivité...), la permanence des enseignables (afin de parer aux changements de contenus trop fréquents et aux effets de mode), la neutralité des enseignables (afin de ne pas favoriser certains élèves en s'adossant aux pratiques de leur milieu)... Mais, d'un autre côté, il lui faut aussi, via la catégorie de pratiques, se donner les moyens de penser la spécificité des pratiques scolaires au regard des pratiques extrascolaires, (et conséquemment, leurs relations et les effets possibles de celles-ci), ainsi que les intérêts possibles de leur diversification, de leur

\footnotetext{
${ }^{23}$ Voir Oudart 2001 qui montre l'importance de la répétition dans les réponses aux lettres de réclamation dans les entreprises de vente par correspondance.

${ }^{24}$ Voir, dans ce numéro, les contributions de C. Boré et C. Masseron.
} 
évolution et de leurs variations selon les apprenants. Cette tension peut encore permettre d'éclairer d'un autre jour les débats autour des situations d'écriture (plus ou moins «fonctionnelles »), des genres d'écrits sollicités (rédactions, dissertations, écrits longs...), des positions convoquées (d'invention, de commentaire...). Ainsi encore, la tension entre une conception «individuelle» et une conception «collective» de l'écriture peut être constituée, dans le champ théorique de la didactique, comme un axe de variation pertinent pour spécifier:

- les représentations et les pratiques sur lesquelles se fonde l'enseignement ainsi que les pratiques sociales qu'il constitue comme objectifs possibles ;

- les opérations qu'il pose comme spécifiques ou fondamentales dans l'écriture ou dans son enseignement (par exemple, dans le cadre d'une conception «individuelle», il s'agit plutôt des opérations de textualisation et de scription, de surcroît essentiellement envisagées dans leurs aspects syntaxiques et orthographiques) ;

- les dispositifs d'enseignement (individuels/collectifs) mis en place et la fonction qui leur est attribuée.

Dans cette perspective, la contradiction interthéorique devient une tension structurante du modèle de didactique de l'écriture...

Ainsi enfin, le débat autour de l'attitude réflexive, me semble pouvoir être constitué didactiquement comme la nécessité de penser conjointement - et donc d'articuler - deux types de contraintes :

- une contrainte structurellement liée aux compétences et à leur enseignement (scolaire) : la compétence scripturale, comme toute autre compétence, pouvant être définie comme capacité d'effectuation et comme capacité d'analyse et d'évaluation du faire; la distance réflexive, étant posée de surcroît comme une nécessité pour construire l'écriture comme objet d'enseignement (de discussion, d'analyse, d'évaluation...),

- une contrainte structurellement liée aux variations socio-culturelles dans les modes de construction, les usages et les valeurs attachées à l'attitude réflexive.

Conséquemment, cela impose de traiter des questions plus « fines » qui portent, par exemple, sur les effets possibles de variations dans l'intervention concernant :

- les domaines sur lesquels porte la réflexion («formels» ou pragmatiques); - la manière dont la démarche réflexive s'appuie ou non sur les questions, les représentations, les critères, le vocabulaire... des élèves ;

- son articulation avec les pratiques mises en place (avec, par exemple, la question d'un réinvestissement plus ou moins différé de ce qui a été analysé) ;

- l'imposition ou la coconstruction des notions en jeu...

Dans tous ces cas de figure, il me semble ainsi que la didactique n'a pas à «trancher» entre des thèses différentes, voire opposées, qui ont chacune leur pertinence dans le cadre théorique qui est le leur, mais qu'il lui appartient de les instaurer comme tensions heuristiques dans l'espace théorique qui lui est propre pour préciser ses questions, analyser les fonctionnements mis en place et les problèmes qu'ils suscitent, fonder et évaluer d'autres fonctionnements possibles. 


\section{De quelques considérations méthodologiques}

Les propositions avancées en matière de relations entre disciplines s'articulent à des positions méthodologiques qui expliquent, au moins en partie, les problèmes soulevés et peuvent permettre de préciser encore les modes de collaboration possibles entre disciplines. De ce point de vue, j'avancerai très succinctement quelques caractéristiques qui me paraissent attachées à l'approche didactique de l'écriture.

\subsection{L'attention accordée aux écrits des élèves}

En premier lieu, je dirais que, fondamentalement, la didactique s'intéresse aux produits réalisés par les élèves. Cette attention est emblématique de la perspective didactique : on imagine mal en effet, dans cette discipline, des recherches sur l'écriture n'étudiant pas les écrits ou ne s'y référant pas. Cela ne signifie d'ailleurs pas que la didactique ne prenne pas en compte d'autres dimensions ou d'autres données (cf. 4.3). Cela rend simplement compte du fait que ces autres dimensions ne prennent sens qu'en relation avec les écrits envisagés comme traces des effets de l'enseignement, des apprentissages, du développement de la compétence scripturale, des problèmes qu'elle rencontre (cf. 4.2).

L'analyse de ces écrits, en tant qu'elle est nodale, s'articule sans doute à trois impératifs, conjoints ou disjoints selon les études :

- l'intensivité de l'analyse, c'est-à-dire la prise au sérieux ${ }^{25}$ du texte réalisé, nécessitant la mise en place de catégories aussi précises que possibles ;

- l'extensivité diachronique, c'est-à-dire la mise en histoire de ces écrits par rapport à d'autres écrits réalisés soit par les élèves considérés, soit par d'autres, plus jeunes ou plus âgés, afin de situer les produits analysés dans une histoire individuelle ou collective ;

- l'extensivité synchronique, c'est-à-dire la mise en relation de ces écrits avec d'autres écrits, réalisés par les élèves considérés ou par d'autres, permettant d'inscrire les performances dans un éventail de possibles et de les comparer afin de préciser les consonances ou dissonances dans le développement de la compétence scripturale.

\subsection{Une description orientée}

Les descriptions d'écrits effectuées dans une perspective didactique me paraissent orientées par quelques principes importants qui modèlent les formes d'attention mentionnées précédemment. J'évoquerai très succinctement trois d'entre eux parmi les plus déterminants.

Le premier concerne la relation au contexte ${ }^{26}$. Il s'agit en fait de considérer l'écrit en tant qu'il constitue une réponse, une manière de faire avec (ou contre) des

\footnotetext{
25 J'entends par cette expression la mise en œuvre de méthodes et de catégories aussi « fines » que celles utilisées pour l'analyse des écrits « légitimes » (Voir Fabre Cols, éd, 2000).

${ }^{26}$ Voir aussi, sur l'importance de cette dimension, les contributions de J.P. Bernié et M.C. Elalouf, dans ce numéro.
} 
contraintes structurant une situation. En d'autres termes, la didactique tend à considérer que le produit d'une activité peut difficilement se comprendre, s'analyser et s'évaluer en dehors d'un contexte qui le modèle. De ce point de vue, les situations scolaires d'écriture ne sont donc pas considérées comme un habillage, voire comme une variable parasite, mais sont définitoires de l'activité et de ses résultats. Cela implique complémentairement de spécifier les genres d'écrits afin de ne pas généraliser en parlant, par exemple, d'analyse de la compétence narrative sans plus de précaution, lorsqu'il s'agit d'analyse d'une performance, dans une situation singulière, sollicitant telle catégorie de récit, dans tel genre, scolaire ou scolarisé...

Cela implique, complémentairement encore, que la didactique privilégie les situations dites écologiques (de classe ou possiblement de classe ${ }^{27}$ ) pour trois raisons au moins :

- elles sont au cœur du domaine didactique en tant qu'il concerne l'enseignement-apprentissage scolaire et son évaluation ;

- les formes de ces situations sont celles que la didactique travaille en considérant qu'elles modèlent les performances ;

- leur analyse permet de penser non seulement les variations de ces performances mais encore leurs relations aux situations et aux performances dans d'autres disciplines et en dehors de l'école.

Ce principe impose en tout cas à la didactique, et ce n'est pas là un mince défi, de construire les moyens méthodologiques de penser la complexité28.

Le second principe qui oriente la description des écrits des élèves - absolument lié à la question du contexte - concerne leur mise en relation avec l'enseignement et les apprentissages dans leur dimension diachronique.

Ainsi, ce qui importe principalement à un didacticien est de pouvoir mieux appréhender comment fonctionnements et dysfonctionnements réfèrent aux apprentissages - eux mêmes reliés à l'enseignement - en ce qu'ils sont des traces d'activités cognitives, d'accélérations, de ralentissements, de régressions, d'obstacles, de déséquilibres...

Ces spécificités placent nécessairement les questions associées à la temporalité au cœur de la réflexion didactique: temps de l'enseignement, temps des apprentissages, moments stratégiques de sollicitation des productions des élèves...

Le troisième principe, tout aussi fortement associé aux précédents, consiste en l'articulation, dans l'analyse des écrits des élèves, des fonctionnements et des dysfonctionnements (sachant que ces deux catégories sont fondamentales à définir dans une perspective didactique). En effet, c'est cette articulation (et non simplement l'un ou l'autre des deux pôles), qui permet de «configurer» un état possible de la compétence en pensant d'un côté ce qui est «fixe » ou «installé », en progression ou en régression, automatisé ou en questions..., en pensant d'un autre côté ce qui pose

\footnotetext{
27 À la différence des situations dites de laboratoire...

${ }^{28}$ Et non, à l'instar d'autres disciplines, de tenter de la réduire. Sur cette question le débat est d'ailleurs compliqué et nécessiterait un article à lui seul. De surcroit, la position ici avancée n'empêche nullement d'envisager des articulations entre situations «écologiques» et de «laboratoire».
} 
plus ou moins problème dans l'activité scripturale et les objets langagiers euxmêmes...

\subsection{Le croisement des modes de recueil et de traitement des données}

Mon insistance sur l'importance des écrits, voire leur caractère emblématique dans une perspective didactique, doit cependant être relativisée par la prise en compte des croisements méthodologiques.

Ces croisements me semblent nécessités par cinq facteurs :

- le fait que l'objectif est de développer la compétence scripturale la plus « large $»^{29}$ possible (dans cette perspective, les écrits ne constituent que des traces très imparfaites de la production et, de telle nature qu'il ne permettent pas véritablement d'expliquer nombre de phénomènes structurant les produits);

- le mode de construction de la compétence qui intègre savoirs, opérations, rapport à la pratique (Barré de Miniac 2000, Pratiques 2002) et nécessite donc, pour saisir l'écriture, outre l'analyse des produits, celle des observations et des déclarations ; - l'importance d'une approche des logiques des scripteurs-apprenants, nécessitant ici encore observations et déclarations sollicitées, envisagées de surcroît au travers d'un prisme compréhensif susceptible de limiter, autant que faire se peut, les risques de l'ethnocentrisme ou de l'« adultocentrisme »;

- la nécessité de préciser le contexte des tâches aussi finement que possible ainsi que la manière dont il a été lui-même reconstruit et interprété par les sujets (cf. 4.2) ;

- l'interdisciplinarité structurelle de la didactique (cf. 2.3), qui lui permet d'éprouver diverses hypothèses quant aux fonctionnements - dysfonctionnements et à leurs sources possibles.

J'ai bien conscience, au moment de conclure cet article, d'avoir été elliptique sur de trop nombreux points ( les modèles didactiques de l'écriture, les concepts spécifiques...). Je souhaite cependant avoir contribué, ne fût-ce que très modestement, à mieux faire comprendre ce qu'est la didactique, comment elle aborde les questions liées à l'écriture, comment elle peut entrer en dialogue avec les autres disciplines qui s'y intéressent... A partir de là, il me reste à espérer que de nouvelles recherches contribueront à dissiper les malentendus possibles et à manifester les intérêts de confrontations interdisciplinaires, dans le respect mutuel.

\footnotetext{
${ }^{29}$ Traductible dans le maximum de champ de pratiques possibles.
} 


\section{BIBLIOGRAPHIE}

BARRÉ De Miniac C. (2000) Le rapport à l'écriture. Aspects théoriques et didactiques, Villeneuve d'Ascq, Presses Universitaires du Septentrion.

Barré DE Miniac C. ed (2000) Copie et modèle : usages, transmission, appropriation de l'écrit, Paris, I.N.R.P.

Borzeix A., Fraenckel B. eds (2001) Langage et travail. Communication, Cognition, Action, Paris, CNRS Editions.

BRASSART D.G., REUTER Y. (1992) «Former des maittres en français. Eléments pour une didactique de la didactique du français », Etudes de linguistique appliquée, $\mathrm{n}^{\circ}$ 87, Recherches en didactique du français et formation des enseignants, juillet-septembre, pp : 11-24.

CheVallard Y. (1985) La transposition didactique, Grenoble, La pensée sauvage.

DABÈnE M. (1987) L'adulte et l'écriture. Contribution à une didactique de l'écrit en langue maternelle, Bruxelles, De Boeck-Wesmael.

Daunay B. (2002) La paraphrase dans l'enseignement du français, Berne, Peter Lang.

DelCambre P. (1997) Ecriture et communications de travail. Pratiques d'écriture des éducateurs spécialisés, Villeneuve d'Ascq, Presses Universitaires du Septentrion

De Pietro J.F., Schneuwly B. (2003) «Le modèle didactique du genre : un concept de l'ingénierie didactique », Les cabiers THEODILE, $\mathrm{n}^{\circ} 3$, Villeneuve d'Ascq.

Dolz J., Scheuwly B. (1998) Pour un enseignement de l'oral. Initiation aux genres formels à l'école, Paris, ESF.

Enjeux (1996/1997) Vers une didactique de l'oral ?, n 39-40, décembre/mars.

FABRE-Cols C. ed (2000) Apprendre à lire des textes d'enfants, Bruxelles, De Boeck-Duculot.

HALtÉ J.F. (1992) La didactique du français, Paris, PUF, coll. Que sais-je ?

LAHIRE B. (1993) Culture écrite et inégalités scolaires, Lyon, PUL.

LIDIL (1990) Des écrits (extra) ordinaires, $\mathrm{n}^{\circ} 3$, septembre.

OUdART M.C. (2001) Les chargé(e)s de relation clientèle face à la lettre de réclamation, Villeneuve d'Ascq, Presses Universitaires du Septentrion.

Penloup M.C. ( 1999) L'écriture extrascolaire des collégiens. Des constats aux perspectives didactiques, Paris ESF.

Pratiques (2002) Images du scripteur et rapports à l'écriture, $\mathrm{n}^{\circ} 113-114$, juin.

Repères (2001) Les pratiques extrascolaires de lecture et d'écriture des élèves, $\mathrm{n}^{\circ} 23$.

Reuter Y. (1994 a) «La didactique du français. Propositions », Inforec, n 16, octobre, pp 1724.

Reuter Y., (1994 b) «A propos des relations dysfonctionnements - causes - remédiations dans l'évaluation », Recherches, n 21, Pratiques d'évaluation, pp : 147-159. 
Yves Reuter

REUTER Y. (1996) Enseigner et apprendre à écrire, Paris, ESF.

REuter Y. (1998) «De quelques obstacles à l'écriture de recherche », Lidil, n 17, Pratiques de l'écrit et des modes d'accès au savoir dans l'enseignement supérieur, pp : 11-23.

Reuter Y. (2000) La description. Des théories à l'enseignement-apprentissage, Paris, ESF.

REUTER Y. (2001) «Eléments de réflexion à propos de l'élaboration conceptuelle en didactique du français », dans Marquillo M., ed: Questions d'épistémologie en didactique du français, Poitiers, Université de Poitiers - Cabiers Forell, pp : 51-66.

REUTER Y. (2002) «Quelques questions à propos des formalisations de l'écriture en didactique du français », Pratiques, n 115-116, L'écriture et son apprentissage, décembre, pp : 29-36.

REuTER Y. (2003) «La construction de la lecture en didactique », dans Egger E., ed : Mobiles et mouvements pédagogiques, Neuchâtel, IRDP, pp : 37-43.

REUTER Y. éd (1994) Les interactions lecture-écriture, Berne, Peter Lang.

REUTER Y. éd (1998) La description. Théories, recherches, formation, enseignement, Villeneuve d'Ascq, Presses Universitaires du Septentrion. 